\title{
Measurement of the dynamic phase profile of transparent fluids
}

\section{Medición del perfil dinámico de fase de fluidos transparentes}

\author{
N. I. Toto-Arellano $\left(1,{ }^{*}, A\right)$, D. I. Serrano-García(2,A), A. Martínez-García ${ }^{(2, A)}$, \\ G. Rodríguez-Zurita( ${ }^{(3, A)}, A$. Montes-Pérez ${ }^{(2, A)}$, J. M. Miranda-Gómez ${ }^{(1)}$, \\ G. Resendiz-López ${ }^{(1)}$, A. González Rosas(1), L. García Lechuga(1), G. A. Parra Escamillaa(1,A) \\ 1. División de Óptica y Fotónica, Área de Electromecánica Industrial, Universidad Tecnológica de Tulancingo, \\ Hidalgo, México. \\ 2. Centro de Investigaciones en Óptica A.C, León, Guanajuato, México. \\ 3. Benemérita Universidad Autónoma de Puebla, Puebla, México. \\ ${ }^{(*)}$ Email: ivantotoarellano@hotmail.com \\ A: miembro de AMO / AMO member \\ Recibido / Received: 17/07/2012. Revisado / Revised: 17/01/2013. Aceptado / Accepted: 22/01/2013. \\ DOI: http://dx.doi.org/10.7149/0PA.46.1.21
}

\begin{abstract}
:
This communication describes some details of modulation of polarization which are useful in phaseshifting interferometry applied to the study of transparent samples. As an application, the case of a two-beam phase-grating interferometer is discussed on the grounds of polarization analysis as an example. This system does not require micro-polarizer arrays or special software to isolate external vibrations; it uses a double window, and generates two beams whose separation can be varied according to the characteristics of the grid used. Experimental results are also given.
\end{abstract}

Key words: Interferometry, Phase shifting, Polarization, Transparent samples.

\section{RESUMEN:}

En este trabajo se describen técnicas de modulación en polarización que son útiles en interferometría de corrimiento de fase aplicada a la medición de la fase dinámica fluidos transparentes. Como una aplicación se discute el caso un interferómetro de doble ventana con rejilla de fase que usa polarización. La configuración presentada no requiere de micro-polarizadores o de paquetería computacional adicional para eliminar ruido causado por vibraciones, este sistema no usa una doble ventana, es capaz de generar dos haces cuya separación puede ser variada de a cuerdo a las características de la rejillas usada. Se presentan los resultados experimentales obtenidos.

Palabras clave: Interferometría, Corrimiento de Fase, Polarización, Objetos Transparentes.

\section{REFERENCIAS Y ENLACES / REFERENCES AND LINKS}

[1]. H. Zhang Hu, " Polarization heterodyne interferometry using a simple rotating analyzer.1: Theory and error analysis", Appl. Opt. 22, 2052-2056 (1983). DOI

[2]. J. P. Liu, T. C. Poon, "Two-step-only quadrature phase-shifting digital holography", Opt. Lett. 34, 250252 (2009). DOI

[3]. I. Yamaguchi, T. Zhang, "Phase-shifting digital holography", Opt. Lett. 22, 1268-1270 (1997). DOI

[4]. I. Yamaguchi, J. Kato, S. Ohta, J. Mizuno, "Image formation in phase-shifting digital holography and applications to microscopy", Appl. Opt. 40, 6177-6186 (2001). DOI

[5]. S. Nakadate, H. Saito, "Fringe scanning speckle-pattern interferometry", Appl. Opt. 24, 2172-2180 (1985). DOI

[6]. J. A. Ferrari, E. M. Frins, C. D. Perciante, "A new scheme for phase-shifting ESPI using polarized light", Opt. Commun. 202, 233-237 (2002). DOI 
[7]. J. H. Bruning, D. R. Herriott, J. E. Gallagher, D. P. Rosenfeld, A. D. White, D. J. Brangaccio, “Digital wavefront measuring interferometer for testing optical surfaces and lenses", Appl. Opt. 13, 26932703 (1974). DOI

[8]. W. D Griffin, "Phase-shifting shearing interferometer", Opt. Lett. 26, 140-141 (2001). DOI

[9]. M. P. Kothiyal, C. Delisle, "Shearing interferometer for phase shifting interferometry with polarization phase shifter", Appl. Opt. 24, 4439-4442 (1985). DOI

[10]. N. I. Toto-Arellano, D. I. Serrano-García, A. M. García, G. R. Zurita, A. Montes-Pérez, "4D profile of phase objects through the use of a simultaneous phase shifting quasi-common path interferometer", J. Opt. 13, 115502 (2011). DOI

[11]. D. I. Serrano-García, N. I. Toto-Arellano, A Martínez-García, J. A. R. Álvarez, G. R. Zurita, “Dynamic phase profile of phase objects based in the use of a quasi-common path interferometer", Optik 123, 1742-1745 (2012). DOI

[12]. M. Novak, J. Millerd, N. Brock, M. North-Morris, J. Hayes, J. Wyant, “Analysis of a micropolarizer arraybased simultaneous phase-shifting interferometer", Appl. Opt. 44, 6861-6868 (2005). DOI

[13]. B. Barrientos-García, A. J. Moore, C. Pérez-López, L. Wang, T. Tschudi, "Transient deformation measurement with electronic speckle pattern interferometry by use of a holographic optical element for spatial phase stepping", Appl. Opt. 38, 5944-5947 (1999). DOI

[14]. B. Barrientos-García, A. J. Moore, C. Pérez-López, L. Wang, T. Tschudi, "Spatial phase-stepped interferometry using a holographic optical element", Opt. Eng. 38, 2069-2074 (1999). DOI

[15]. N. I. Toto-Arellano, G. Rodriguez-Zurita, C. Meneses-Fabian, J. Vázquez-Castillo, C. Robledo-Sánchez, "Phase shifts in the Fourier spectra of phase gratings and phase grids: an application for one-shot phase-shifting interferometry", Opt. Express 16, 19330-19341 (2008). DOI

[16]. D. Malacara, M. Servin, Z. Malacara, Phase Detection Algorithms in Interferogram Analysis for Optical Testing, Chap. 6, Wiley, New York (2005). DOI

[17].C. Ghiglia, M. D. Pritt, Two-Dimensional Phase Unwrapping: Theory, Algorithms and Software John Wiley \& Sons, New York (1998).

[18]. A. Dávila, D. Kerr, G. H. Kaufmann, OPTOFRINGE, v.1, software developed by A. Dávila and G. Mendiola-Anda, CIO, León, Gto, México (1998).

[19]. J. C. Wyant, “Dynamic interferometry”, Opt. Photon. News 14, 36-41 (2003). DOI

\section{Introduction}

Modulation of polarization is often used in optical interferometry [1], digital holography [2-4], ESPI and shearography [5-8] because it allows one to analyze samples using non-contact techniques with high accuracy. The use of phase shifting modulated by polarization has the advantage of not requiring mechanical components, such as a Piezoelectric Transducer (PZT), to obtain the desired phase shifts. A common optical system uses linear polarizing filters and birefringent quarter-wave plates to achieve modulation [9-11]; in some situations, exact retardation can not be used, and certain considerations have to be taken into account to extract the correct optical phase data.

In this paper, we propose an optical system that uses single-shot phase shifting interferometry techniques modulated by polarization, allowing it to show no sensitivity to external vibration, since the two beams produce a common path interferometer, which allows one to measure dynamic events with high accuracy; The configuration presented does not require expensive items such as micropolarizers [12], only conventional polarizers, nor does it need additional software to eliminate noise caused by vibration, as the two beam interferometer is stable. Analysis and discussion about non-exact quarter-wave retardation are presented and corrected to obtain a desired phase shift between interfering beams; this analysis allows the use of retardants that do not operate on the same wavelength of the laser used, and it emerges as a necessity if you do not have the necessary optical elements. Implementing a two beam phase grating Mach Zehnder Interferometer (TBPGMZI) modulated by polarization, experimental results for static and dynamic events for thin-transparent samples are presented. 


\section{Experimental setup}

A combination of a quarter-wave plate $Q$ and $a$ linear polarizing filter $\mathrm{P}_{0}$ generates linearly polarized light oriented at $\pm 45^{\circ}$ when entering the Mach Zehnder (MZ) interferometer from a $\mathrm{YVO}_{3}$ laser operating at $532 \mathrm{~nm}$ (see Fig 1). This configuration generates two symmetrically displaced beams by moving mirrors $M$ and $M^{\prime}$, enabling one to change the spacing $x_{0}$ between beam centers. Two retardation plates $\left(Q_{L}\right.$ and $Q_{R}$ ), with mutually orthogonal fast axes, are placed in front of the beams (A, B) to generate left and right nearly-circular polarized light [11-14].

The transparent sample is collocated on $\mathrm{B}$, and $A$ is used as a reference beam. A phase grid carefully constructed by superposing two commercially available phase gratings with their respective grating vectors at $\pm 90^{\circ}$ is placed at the system's Fourier planes as the pupil. As shown in figure 1 , placing a grating of spatial period $d=\lambda f / x_{0}$ on the Fourier plane, where $\lambda$ is the wavelength and $f$ the focal length. The corresponding transmittance is given by $G(\mu, v)$. The image $O^{\prime}(x, y)$ formed by the system consists basically of replications of each window at distances $X_{0}$; that is, the convolution of $O(x, y)$ with the Fourier transform of the phase grating.
Taking the rulings of one grating along the $\mu$ direction, and the rulings of the second grating along the $v$ direction, the resulting centered phase grid can be written as:

$$
\begin{aligned}
G(\mu, v)= & e^{i 2 \pi A_{g} \sin \left[2 \pi X_{0} \mu\right]} e^{i 2 \pi A_{g} \sin \left[2 \pi X_{0} \nu\right]}= \\
= & \sum_{q=-\infty}^{\infty} J_{q}\left(2 \pi A_{g}\right) e^{i 2 \pi \cdot q X_{0} \mu} \times \\
& \times \sum_{r=-\infty}^{\infty} J_{r}\left(2 \pi A_{g}\right) e^{i 2 \pi \cdot r X_{0} v},
\end{aligned}
$$

where the period along each axis directions are taken as $X_{0}$, with $2 \pi A_{g}$ being the grating phase amplitude, and $J_{q}$ and $J_{r}$ the Bessel functions of the first kind of integer order $q, r$, respectively. The Fourier transform of the phase grid becomes:

$$
\begin{array}{r}
\widetilde{G}(x, y)=\sum_{q=-\infty}^{q=\infty} \sum_{r=-\infty}^{r=\infty} J_{q}\left(2 \pi A_{g}\right) J_{r}\left(2 \pi A_{g}\right) \times \\
\times \delta\left(x-q X_{0}, y-r X_{0}\right),
\end{array}
$$

which consists of point-like diffraction orders distributed on the image plane at the nodes of a lattice with a period given by value $X_{0}$.

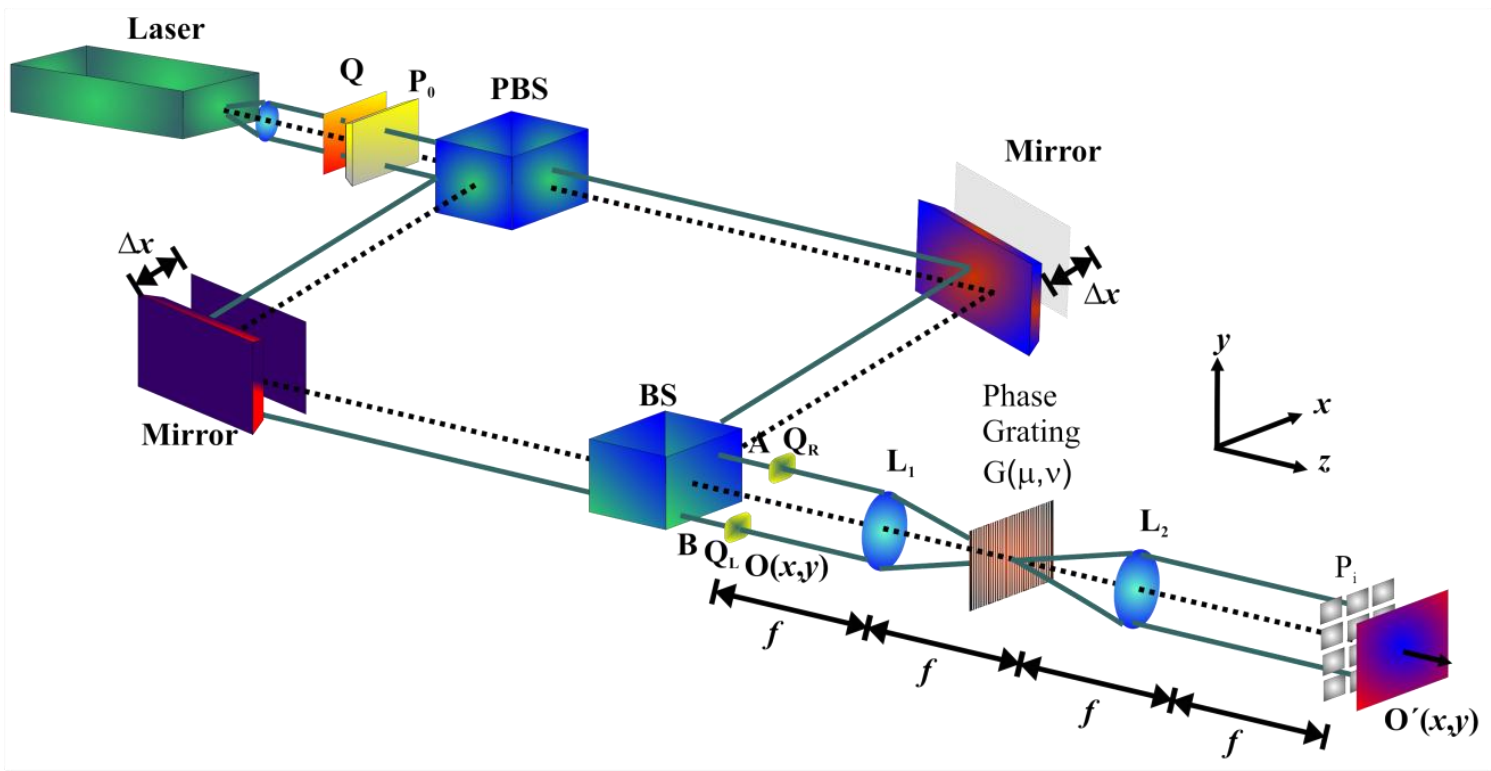

Fig. 1. Simultaneous phase shifting quasi-common path interferometer with modulation of polarization. PBS: Polarizing beam splitter. BS: Beam splitter. $\Delta x$ : Mirror displacement. $O(x, y)$ : Object plane. $O^{\prime}(x, y)$ : Image plane. $L_{i}$ Lens. $G(\mu, v)$ : Phase grid. $P_{0}$ and $P_{i}$ : Polarizers. $Q, Q_{L}$ and $Q_{R}$ : $Q$ uarter wave plate. Grating period, $d=110$ lines $/ \mathrm{mm}$. $f=150 \mathrm{~mm}$. 


\section{Fringe modulation and contrast in phase grating interferometry}

Phase grid interferometry is based on a two crossed phase grating placed as the pupil in a 4 -f Fourier optical system. A convenient window pair for a grating interferometer implies a vectorial amplitude transmittance given by:

$$
\vec{O}(x, y)=\vec{J}_{L} \cdot A\left(x-\frac{x_{0}}{2}, y\right)+\vec{J}_{R} B\left(x+\frac{x_{0}}{2}, y\right)
$$

where $x_{0}$ is considered as the mutual separations between the centers of each window along the coordinate axis, with an arbitrary retardation $\alpha^{\prime}$ and the Jones vectors, $\vec{J}_{L}$ and $\vec{J}_{R}$, defined as:

$$
\vec{J}_{L}=\left(\begin{array}{c}
1 \\
e^{i \alpha^{\prime}}
\end{array}\right), \quad \vec{J}_{R}=\left(\begin{array}{c}
1 \\
e^{-i \alpha^{\prime}}
\end{array}\right)
$$

One beam aperture can be described as $A(x, y)$, and the second one as $B(x, y)=$ $\exp (i \phi(x, y))$, representing a relative phase between both windows described by $\phi(x, y)$. As shown in Fig. 1, by placing a grating of spatial period $d=\lambda f / X_{0}$ on the Fourier plane, the corresponding transmittance is given by $G(\mu, v)$. The image $\vec{O}^{\prime}(x, y)$ formed by the system consists basically of replications of each window at distances $X_{0}$; that is, the convolution of $\vec{O}(x, y)$ with the Fourier transform of the phase grating. Invoking the condition of matching firstneighboring orders, $X_{0}=x_{0}, q^{\prime}=q+1$ and $r^{\prime}=$ $r$. The image is then basically described by:

$$
\begin{aligned}
& \vec{O}^{\prime}(x, y)=\vec{O}(x, y) * \widetilde{G}(x, y)= \\
& =\vec{J}_{L} \sum_{q, r}^{\infty} J_{q} J_{r} A\left(x-\left(q+\frac{1}{2}\right) x_{0}, y-r x_{0}\right)+ \\
& \quad+\vec{J}_{R} \sum_{q^{\prime}, r^{\prime}}^{\infty} J_{q^{\prime}} J_{r^{\prime}} B\left(x-\left(q^{\prime}+\frac{1}{2}\right) x_{0}, y-r^{\prime} x_{0}\right) \\
& =\sum_{q=-\infty}^{\infty} \sum_{r=-\infty}^{\infty} \vec{J}_{L} J_{q} J_{r}+\vec{J}_{R} J_{q+1} J_{r} e^{\left[i \phi\left(x-\left(q+\frac{1}{2}\right) x_{0}, y-r x_{0}\right)\right]} .
\end{aligned}
$$

By selecting the diffraction term of order $q r$, after placing a linear polarizing filter with the transmission axis at an angle $\psi, \vec{P}_{\psi}$, its irradiance results as being proportional to:

$$
\begin{aligned}
& \left\|\vec{J}_{L}^{\prime} J_{q} J_{r}+\vec{J}_{R}^{\prime} J_{q+1} J_{r} \cdot e^{i \phi\left(x^{\prime}, y^{\prime}\right)}\right\|^{2}= \\
& =A\left(\psi, \alpha^{\prime}\right) \times\left[\left(J_{q} J_{r}\right)^{2}+\left(J_{q+1} J_{r}\right)^{2}+\right. \\
& \left.\quad+2 J_{q} J_{r}{ }^{2} J_{q+1} \cos \left[\xi\left(\psi, \alpha^{\prime}\right)-\phi\left(x^{\prime}, y^{\prime}\right)\right]\right],
\end{aligned}
$$

where $x^{\prime}$ and $y^{\prime}$ are the coordinates on the image plane,

$$
\begin{aligned}
& \vec{P}_{\psi}=\left(\begin{array}{cc}
\cos ^{2} \psi & \sin \psi \cos \psi \\
\sin \psi \cos \psi & \sin ^{2} \psi
\end{array}\right), \\
& \vec{J}_{L}^{\prime}=\vec{P}_{\psi} \vec{J}_{L}, \quad \vec{J}_{R}^{\prime}=\vec{P}_{\psi} \vec{J}_{R},
\end{aligned}
$$

and $A\left(\psi, \alpha^{\prime}\right)$ and $\xi\left(\psi, \alpha^{\prime}\right)$ are defined as:

$$
\begin{gathered}
A\left(\psi, \alpha^{\prime}\right)=1+\sin (2 \psi) \cdot \cos \left(\alpha^{\prime}\right), \\
\xi\left(\psi, \alpha^{\prime}\right)=\tan ^{-1}\left[\frac{\sin \left(\alpha^{\prime}\right)}{\frac{\cot (2 \psi)}{1+\tan (\psi) \cdot \cos \left(\alpha^{\prime}\right)}+\cos \left(\alpha^{\prime}\right)}\right] .
\end{gathered}
$$

Fringe contrast $m_{q r}$ is represented by:

$$
m_{q r}=\frac{2 J_{q} J_{q-1}}{J_{q}^{2}+J_{q-1}^{2}} .
$$

where each fringe modulation depends on the relative phases between the Bessel functions $J_{q}$.

\subsection{2-D Interference patterns generated by diffraction}

The interference patterns are obtained from the interference between the replicas of each beam, centered around each diffraction order. Figure 2(a) presents the replicas of beam A, with right circular polarization, and the replicas of beam $B$, with left circular polarization; each order is superposed depending on separation $x_{0}$ of the beams at the output of the Mach Zehnder (MZ) system. Figure 2(b) presents the interference pattern generated by the interference of contiguous orders $[(-1,-2),(0,1),(+1,0),(+2,+1)]$, where $x_{0} \cong 0.8 \mathrm{~mm}$.

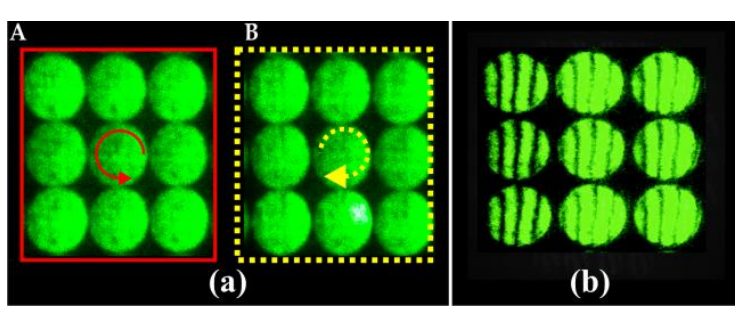

Fig. 2: (a) Replicas of beams A and B. (b) Interference pattern obtained by means of the order diffraction superposition for $x_{0} \cong 0.8 \mathrm{~mm}$. 


\subsection{Phase shifts for inexact retardation}

Figure 3 shows the dependence of retardation $\left(\alpha^{\prime}\right)$ against wavelength $\lambda$ for different birefringent wave plates. This result shows that retardation is not a linear function of wavelength, i.e.: $\alpha^{\prime}(\lambda)=\pi \lambda_{0} / 2 \lambda$, with $\lambda_{0}$ being the operating wavelength of the QWP, and $\lambda$ the wavelength of the laser used; so, the retardation for $\pi / 2$ has to work with the correct wavelength shown in the graph, where the horizontal line shows the case of $\alpha^{\prime}=\pi / 2$. Representing the case of exact quarter-wave retardation, it is readily found that $\xi\left(\psi, \frac{\pi}{2}\right)=2 \psi, A^{2}\left(\psi, \frac{\pi}{2}\right)=1$.

Experimentally, phase shifts result after applying a linear polarizer to each one of the interference patterns generated around each diffracting order at the exit plane $\left(P_{i}\right)$. Each

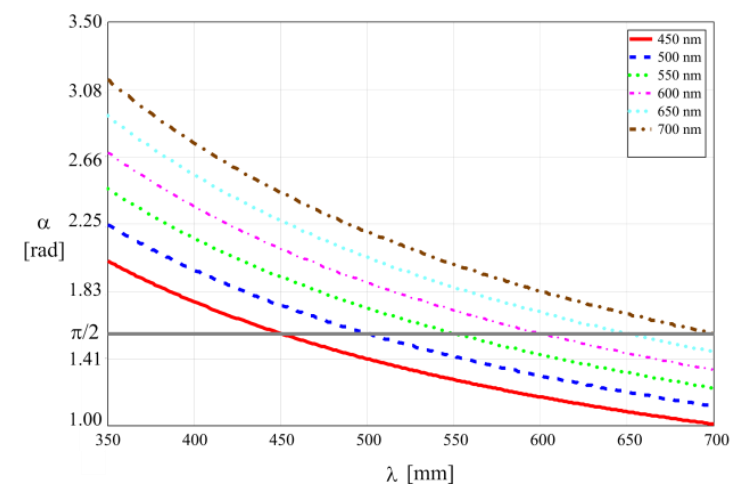

Fig. 3: Dependence of retardation $\left(\alpha^{\prime}\right)$ on wavelength $\lambda$ for different birefringent wave plates.

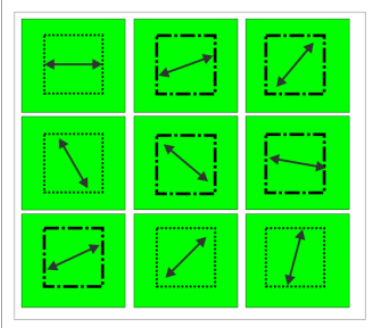

(a)

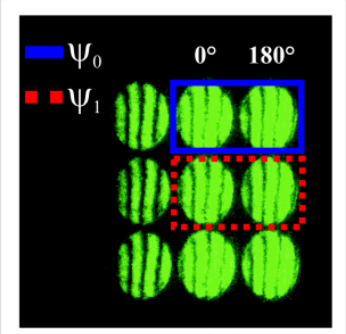

(b)

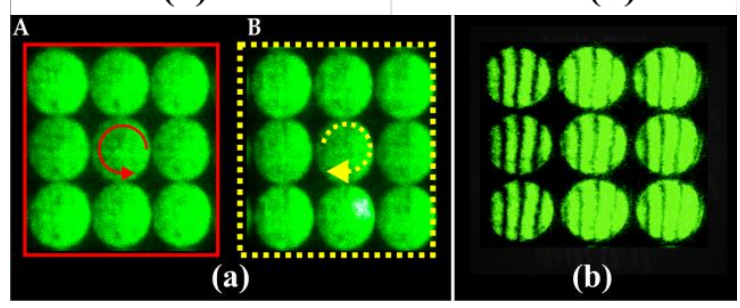

Fig. 4: Replicas of the interference patterns obtained with the phase grid with $\pi$-phase shifts (a) Polarizing filter array. (b) Experimental Interference patterns. polarizing filter transmission axis is adjusted at a different angle $\psi_{i}$ to generate the four phase shifted interferograms. Figure 4(a) shows a representation of the linear polarizers placed over each desired interference pattern. As it can be observed in Fig 4(b), the superposition of replicas of beams A and B obtained through the phase grating used generates interference patterns with a relative phase shift of $\pi$, due to this [15], two phase shifted interferograms can be obtained only using a linear polarizer. This allows a simplification of the polarizing filter array [15]; in this case, only two polarizers $\left(P_{1}\right.$ and $P_{2}$ ) need to be placed, each one covering two patterns with complementary phase shifts. Taking into account the linear polarizer angle corrections due to the different wavelengths used, then $\psi_{0}=0^{\circ}$ and $\psi_{1}=46.577^{\circ}$, which leads to phase shifts $\xi$ of $0, \pi / 2$, and $3 \pi / 2$; this can be seen in Fig. 4(b), where the dotted boxes represent the polarizing filters.

\section{Transparent samples}

A phase object (transparent) that is placed onto beam $\mathrm{B}$ can be expressed as:

$$
O(x, y)=1+i \phi(x, y) .
$$

Using this approach for thin transparent samples, where $|\phi(x, y)|^{2}<<1$, it can be noted that the phase of the object is proportional to the function that defines its shape. This way, we can know the profile of the object analyzing the optical phase; the phase reconstruction is performed using a four step phase shifting algorithm [13-15], so the phase can be obtained from the following equation [16]:

$$
\phi(x, y)=\tan ^{-1}\left(\frac{I_{1}-I_{3}}{I_{2}-I_{4}}\right),
$$

with $I_{i}$ being the intensity measurements captured in a single shot, with the values of $\psi$ given by $\psi_{0}=0^{\circ}, \psi_{1}=46.577^{\circ}$. This result allows us to know the phase profile. Since $n$ interferograms can be obtained simultaneously, the dynamic study of a phase object can be carried out. 


\section{Experimental results}

The phase gratings used are the commercially available ones. They are nominally identical, according to the seller (Edmund Optics' transmission grating, dimensions: $25 \times 25 \mathrm{~mm}^{2}$; dimensional tolerance: $\pm 0.5 \mathrm{~mm}$; substrate: Optical Crown Glass). Under conditions of circular polarization generation, the error in the phase shift $\delta \xi$ is twice the error in the polarizing transmission angle $\delta \psi$; through this, $\delta \xi$ contributes to an error in each sinusoidal pattern intensity, $\delta I$, no greater than $2 \delta \xi=4 \delta \psi$ for the unit fringe contrast. As the values of $\delta \psi$ can be very small, there are no error sources such as nonlinearities of displacements or nonhomogeneities in the measuring field. That way, more flexibility can be used in choosing appropriate algorithms, and the procedure does not require lengthy calibration procedures each time the pattern changes.

The monochromatic camera used is based on a CMOS sensor with $1280 \times 1024$ pixels and with a pixel pitch of $6.7 \mu \mathrm{m}$. Each interferogram was typically composed of arrays of $600 \times 512$ pixels, and then filtered with a conventional low-pass filter to remove sharp edges and details from the image, leaving smooth gradients and lowfrequency details. To reduce differences in irradiance and fringe modulation, each interferogram used was subjected to a rescaling and normalization process, taking its minimum value as the zero gray level, and its maximum value as the 255 gray level. This procedure generates patterns of equal background and equal fringe modulation. For the simultaneous capture of several interferograms, an error source comes from the acquisition of different parts of the same camera used. Other approaches for simultaneous phase shifting interferometry (PSI) have a similar feature; namely, a different sampling of the same distribution. In this procedure, however, no oneto-one correspondence between filter pixels and detection pixels is required because the filter area comprises many pixels. For fringe periods greater than some pixels, this error source does not seem to be greater than the speckle effects. Under these conditions, the remaining error sources are the same as for a typical phaseshifting interferometer. There are several 2D phase unwrapping algorithms, some of them using least squares methods and the branch cut technique[17]; however, for simplicity, the method used for unwrapping the phase data was a non-iterative fast cosine method [18].

A phase step was placed in the path of beam $B$, while beam A was used as a reference; the results obtained are shown in Fig. 5. Figure 5(a) shows the four patterns with $\pi / 2$ phase shifts obtained in a single shot. Figure 5(b) presents the phase profile of the phase object.

Figure 6 shows the case of fluids moved by gravity placed on a microscope slide; this generates phase changes due to the changing layer of the fluid. The upper row of the figure corresponds to oil placed on a microscope slide, while the lower row corresponds to a drop of water moving over the microscope slide.

Figure 7 shows the results for an oil drop placed on a microscope slide; the dynamic evolution of the drop as it goes across the front of the camera can be seen. In this case, small deformations can be observed in the drop,

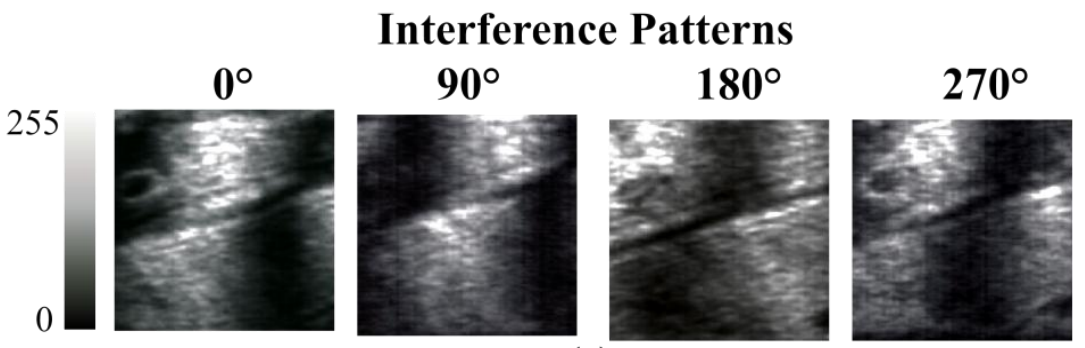

(a)

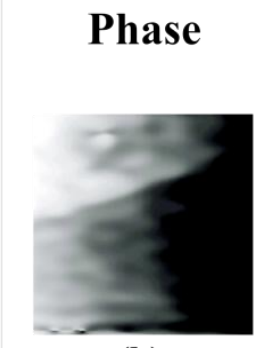

(b)

Fig. 5: Static test objects. (a) Interference patterns. (b) Unwrapped phase. 


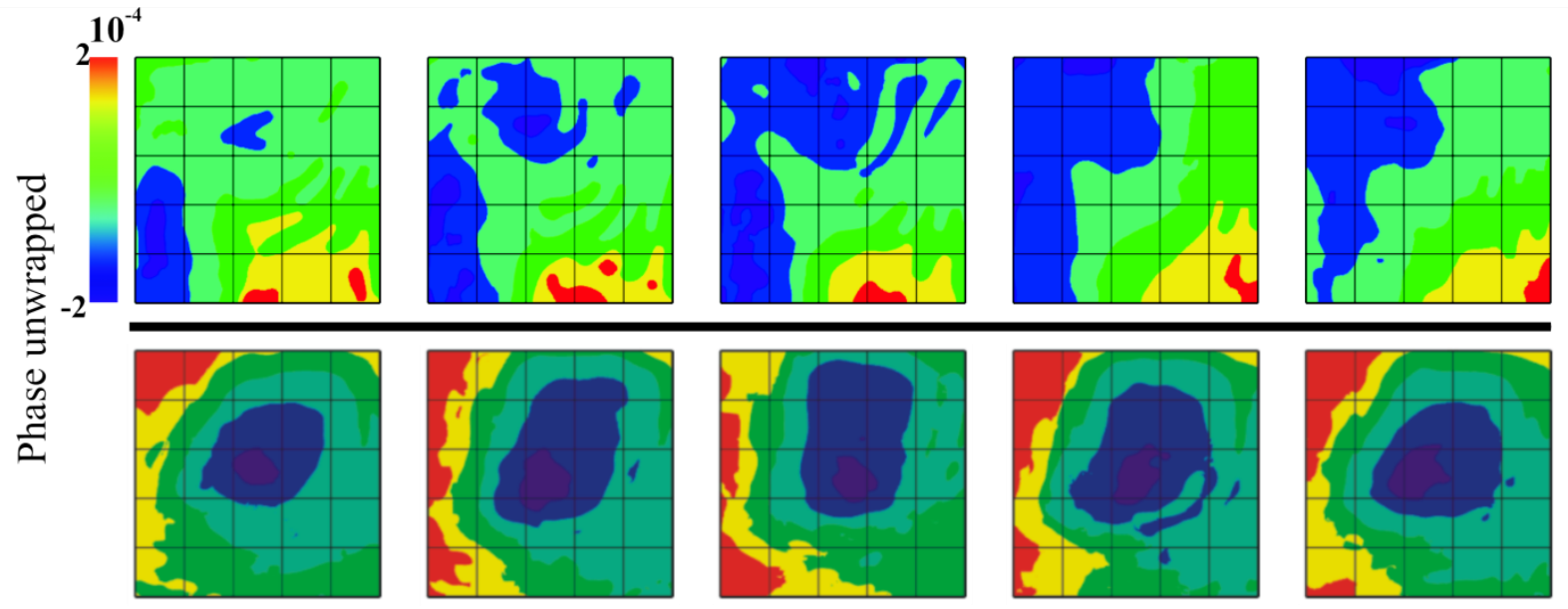

Fig. 6: Dynamics distributions. Evolution of the phase, one capture per second (Representative Frames). Upper row shows oil on a microscope slide. Lower row corresponds to flowing water on a microscope slide.
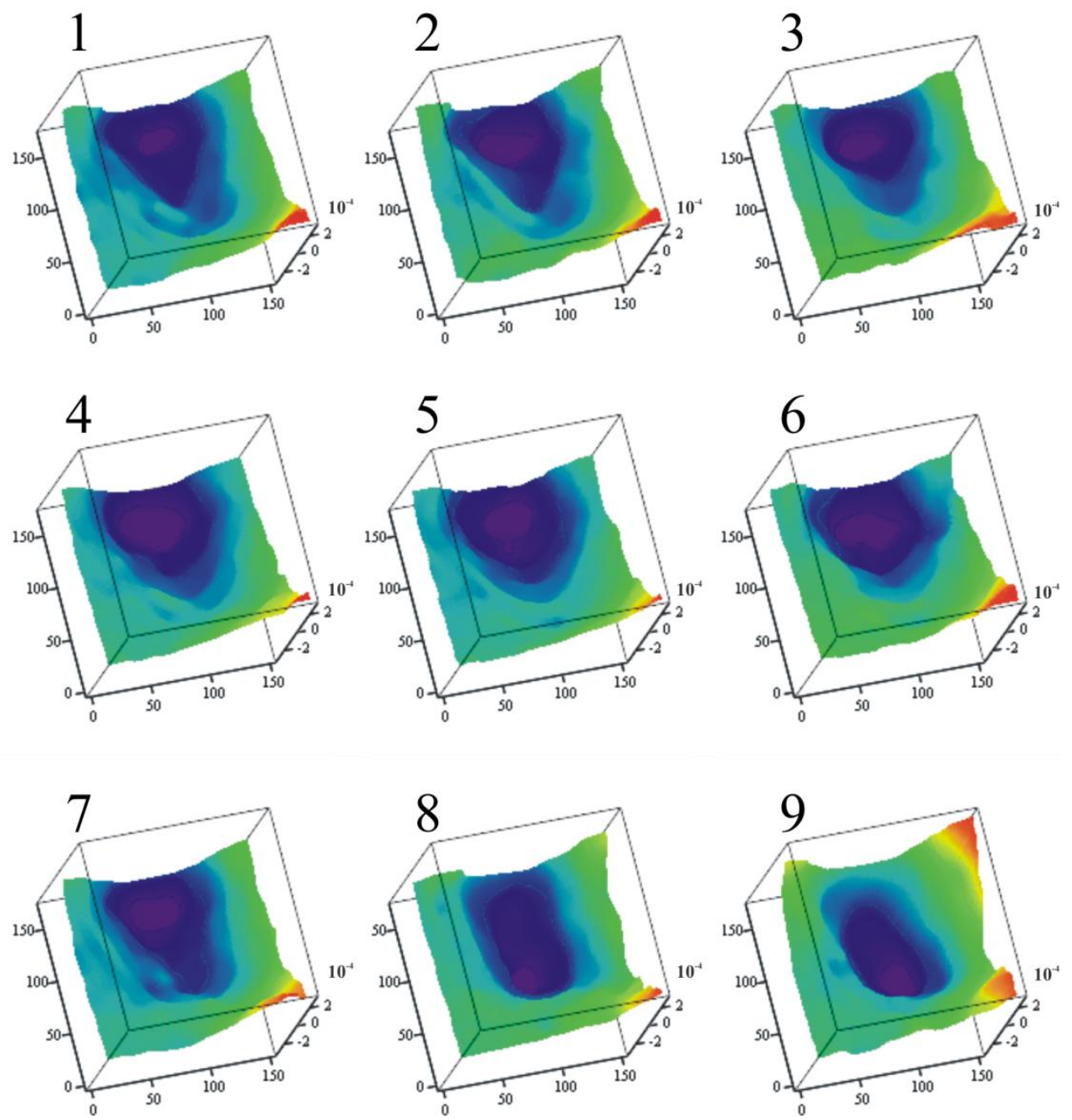

Fig. 7: Immersion oil on a microscope slide. Oil drops moving under gravity on a slide. One capture per 2 second. 
caused by its viscosity and also by the total change effected by gravity. By retrieving a 3D reconstruction of the temporal variation [19], we are also able to observe the change in depth of the oil layer caused by the displacement. These results will allow measuring velocity vector fields of the flow, as well as measuring index refraction changes with the same information retrieved.

\section{Final remark}

The experimental set-up for a polarizing two beam phase grating common-path interferometer based on a Mach Zehnder interferometer has been described to obtain the profile of thin transparent fluids from the analysis of optical phase using phase shifting techniques. This system is able to obtain several interferograms simultaneously. The combinations of the phase shifts generated by the grating simplify the polarizing filter array. This characteristic optimizes the interferometric system used, and allows the analysis of objects of static and dynamic phase.

\section{Acknowledgements}

Authors thank M. A. Ruiz for his contribution in proofreading the manuscript. Enlightening comments and references from anonymous referees are also acknowledged.

Author N.-I. Toto-Arellano expresses sincere appreciation to Luisa, Miguel and Damian for the support provided, and to "Programa de Mejoramiento del Profesorado" (PROMEP) for Grant UTTGO-PTC-016. Partial support from "Sistema Nacional de Investigadores" (SNI) for grant 47446 is also acknowledged.

Author D.-I Serrano-García (Grant: $227470 / 31458$ ) is very grateful to CONACyT for the graduate scholarship granted, and expresses sincere appreciation to Geliztle.

Author A. Montes-Pérez occupies a Postdoctoral position at $\mathrm{CIO}$, and expresses sincere appreciation to CONACyT, for grant 160260/290679-CIO. 